\title{
Le rendez-vous manqué entre Mozart et l'aristocratie parisienne (1778)
}

A Missed "Rendez-vous" between Mozart and the Parisian aristocracy (1778)

David Hennebelle

\section{(2) OpenEdition \\ Journals}

Édition électronique

URL : https://journals.openedition.org/ahrf/13415

DOI : $10.4000 /$ ahrf. 13415

ISSN : 1952-403X

Éditeur :

Armand Colin, Société des études robespierristes

Édition imprimée

Date de publication : 15 février 2015

Pagination : $35-45$

ISBN : 978-2-200-92958-9

ISSN : 0003-4436

Référence électronique

David Hennebelle, «Le rendez-vous manqué entre Mozart et l'aristocratie parisienne (1778) », Annales historiques de la Révolution française [En ligne], 379 | janvier-mars 2015, mis en ligne le 15 février 2018, consulté le 01 juillet 2021. URL : http://journals.openedition.org/ahrf/13415 ; DOI : https://doi.org/ 10.4000/ahrf. 13415 


\title{
LE RENDEZ-VOUS MANQUÉ ENTRE MOZART ET L'ARISTOCRATIE PARISIENNE (1778)
}

David HENNEBELLE

\begin{abstract}
Le troisième séjour de Wolfgang Amadeus Mozart à Paris, entre mars et septembre 1778, a retenu depuis longtemps l'attention des biographes. Si l'on s'accorde généralement pour y voir un tournant majeur dans sa maturation personnelle et artistique, il n'y a guère d'unanimité sur le bilan qu'il convient de dresser de cette expérience et sur les motifs réels de l'échec, échec au demeurant tout relatif au vu des démarches entreprises en un laps de temps relativement court. Le présent article propose d'explorer, en regard avec d'autres carrières musiciennes, une autre piste généralement ignorée : celle de la trop grande brièveté de sa présence parisienne.
\end{abstract}

Mots-clés : Mozart, Paris, aristocratie, patronage musical.

«Va à Paris ! et bientôt. Prends place auprès des grands seigneurs, aut Cesar aut nihil [...]. C'est de Paris que le renom et la gloire d'un homme de grand talent parviennent au monde entier, la noblesse y considère les gens de talent avec la plus grande déférence, estime et courtoisie $»^{1}$.

Ainsi s'exprime Leopold Mozart dans une lettre écrite au début de 1778, peu de temps avant que son fils ne remette le pied sur le pavé parisien. On ne peut qu'être saisi par le contraste entre les promesses du troisième séjour parisien de Mozart - largement fondées sur les souvenirs glorieux des deux premiers - et le gâchis manifeste qui en résulta finalement. Partant

(1) Wolfgang-Amadeus MozART, Correspondance (traduction française de Geneviève Geffray), Paris, Flammarion, 1987, vol. 2, p. 246. Lettre de Leopold Mozart à son fils, Salzbourg, 12 février 1778. 
de ce constat, de nombreuses questions restent pendantes. Mozart était-il en situation de réussir à Paris ? Quelle fut l'attention que la bonne société lui accorda réellement? Son échec fut-il aussi complet qu'on le présente ordinairement? Quelles sont les causes véritables de ce qui, aujourd'hui, nous apparaît davantage comme un rendez-vous manqué ?

Mozart, chaperonné par sa mère, avait quitté Salzbourg en septembre 1777. Au bout de six mois de démarches insistantes afin d'obtenir un congé pour voyager et rechercher une situation plus prestigieuse, Mozart s'était vu signifier sa mise à pied par son employeur, le prince-archevêque de Salzbourg, le comte Hieronymus Colloredo (1732-1812) ${ }^{2}$. Après plusieurs tentatives infructueuses à Munich et à Mannheim, Paris devait constituer l'étape ultime et décisive dans cette quête. Les ressources musicales qu'offrait la ville lumière avaient en effet de quoi lui redonner espoir :

«C'est le seul endroit où l'on peut gagner de l'argent et se faire honneur [...]. Quiconque a écrit quelques opéras à Paris reçoit quelque chose de fixe par an. Et puis il y a le Concert spirituel, l'accadémie des amateurs [...]. Si l'on donne des leçons, l'usage veut que l'on gagne 3 louis d'or pour 12 leçons. On fait ensuite graver en souscription des sonates, trios et quatuors $»^{3}$.

Outre les grandes institutions musicales comme l'Académie royale de musique et le Concert Spirituel, le patronage de l'aristocratie restait le principal mode d'appropriation de la musique et des musiciens et de structuration de la vie musicale. Le règne de Louis XV avait vu s'épanouir de multiples patronages musicaux à Paris et dans les résidences d'Île-deFrance. C'est par centaines que les compositeurs dédiaient leurs œuvres à des personnes de haut rang. Les concerts privés, numériquement les plus importants, constituaient les «sas » de la reconnaissance, de la protection, de l'intercession et parfois de l'attache permanente. Ils formaient aussi des vases communiquants avec la sphère publique, tout en restant parfaitement distincts. La venue de Mozart coïncida avec une phase de recomposition qui allait dans le sens d'une expansion du marché de la musique. Aux patronages individuels, se substituaient ou s'ajoutaient de plus en plus des formes collectives. Les expériences du Concert des Amateurs, la vie musicale

(2) À Salzbourg, Mozart occupait la charge de konzertmeister de la Cour archiépiscopale. À ce titre, il était surtout requis comme violoniste mais devait aussi composer, notamment de la musique religieuse.

(3) Wolfgang-Amadeus MozART, op. cit., vol. 2, p. 153. Lettre de Mozart à son père, Mannheim, 3 décembre 1777. 
des loges maçonniques, en particulier la Société Olympique, les soutiens aristocratiques au concert à bénéfice et aux projets de théâtre lyrique témoignaient de cet élargissement. Les patronages établis de musiciens à demeure cohabitaient avec des formules plus souples de musiciens invités, se mêlant fréquemment à des amateurs ${ }^{4}$. Pour autant, la bonne société parisienne gardait la haute main sur ces initiatives, leur conservant un caractère strictement aristocratique :

«La Société Olympique, la plus brillante de toutes celles du Palais Royal [...] est composée de 200 Messieurs et de 102 Dames de la plus grande distinction. [...] Les membres de cette société portent, dans leurs assemblées, pour marque distinctive, une plaque brodée en paillettes d'argent, formant une lyre entourée de rayons $»^{5}$.

Mozart pouvait se référer à la réussite parisienne de nombreux musiciens allemands, à l'instar de Christoph Willibald Gluck (1714-1787), dont les opéras avaient révolutionné la scène lyrique.

Sur la route qui le menait à Paris, Mozart se trouvait en possession d'une pile impressionnante de lettres que lui avait envoyées son père afin de lui prodiguer ses sages conseils, depuis les aspects matériels et financiers du voyage jusqu'aux règles de la sociabilité aristocratique, en passant par la prise en compte du goût musical parisien. Il possédait aussi une liste de personnalités à rencontrer mais cet annuaire, basé sur les notes de voyage de Leopold, était largement obsolète. Bercé par le souvenir des deux séjours triomphaux de l'enfant prodige en 1763-1764 et 1766, il se trompait lourdement en écrivant : «Voici la liste de nos connaissances à Paris, qui te reverront toutes avec joie $»^{6}$. À la différence aussi des précédents séjours, il ne disposait que d'une lettre de recommandation pour Diderot, absent de Paris, une autre pour d'Alembert et deux pour le comte von Sickingen, envoyé de la cour du Palatinat. C'était plutôt mince, même si ce genre de sésame n'offrait aucune garantie et pouvait au contraire susciter de l'agacement.

Le 23 mars 1778, Mozart et sa mère arrivaient à Paris. Dès le lendemain, le compositeur salzbourgeois se rendit chez le baron Grimm (1723-1807). Personnage influent au carnet d'adresses particulièrement

(4) David Hennebelle, De Lully à Mozart. Aristocratie, musique et musiciens à Paris (XVII ${ }^{e}$ - XVIII ${ }^{e}$ siècles), Seyssel, Champ Vallon, 2009.

(5) Almanach du Palais Royal pour l'année 1786, p. 135-136. 5 février 1778 .

(6) Wolfgang-Amadeus MOZART, op . cit., vol. 2, p. 231. Lettre de Leopold à son fils, Salzbourg, 
fourni, familier des Encyclopédistes et des salons, il était d'abord connu pour ses articles dans la Correspondance littéraire, philosophique et critique adressée à un souverain d'Allemagne, publiée entre 1753 et 1790 . À plusieurs reprises par le passé, il avait aidé la famille Mozart. Pressé par les lettres de Leopold, il s'engagea sans grand enthousiasme à aider son jeune compatriote : «Je suis accablé d'affaires et d'écritures [...] mais lorsque M. votre fils sera ici, il sera mon secrétaire $»^{7}$. Il commença par lui fournir une lettre de recommandation pour le duc et la duchesse de Rohan-Chabot afin de renouer avec la cousine du roi, la duchesse de Bourbon, qui, petite fille, avait dédié un rondeau pour clavier au jeune virtuose. Cette fois pourtant la magie n'opéra plus et la visite tourna court :

«[...] la D. Chabot arriva et me pria avec la plus grande amabilité de me satisfaire du piano qui était là, du fait qu'aucun des siens n'était en état [...]. Puis elle s'assit et commença à dessiner pendant toute une heure, en Compagnie d'autres messieurs [...].

Ainsi, j'ai eu l'honneur d'attendre une heure entière [...]. Et je ne savais que faire, si longtemps, de froid, de mal de tête et d'ennui. [...] Finalement, pour être bref, je jouai sur ce misérable affreux Pianoforte. [...] je dus donc jouer pour les fauteuils, les tables et les murs. Dans ces conditions aussi abominables, je perdis patience, - je commençai les variations de Fischer, en jouai la moitié et me levai. Il y eut une foule d'Eloges. [...] Donnezmoi le meilleur piano d'Europe mais comme auditeurs, des gens qui n'y comprennent rien, ou qui ne veulent rien y comprendre, et qui ne sentent pas avec moi ce que je joue, j'y perds tout plaisir. [...] les gens font certes des compliments, mais qui s'arrêtent là. Ils me demandent de revenir tel ou tel jour, je joue et ils disent : O c'est un Prodige, c'est inconcevable, c'est étonnant. Et là-dessus addieu $[\ldots] »^{8}$.

Dès le début du séjour, Mozart entra en relation, par l'entremise du flûtiste alsacien Johann-Baptist Wendling côtoyé à Mannheim, avec le duc de Guines (1735-1806), une des figures d'amateur les plus accomplies de son temps. Ayant entamé une brillante carrière militaire et diplomatique, il avait été ministre plénipotentiaire à la cour de Frédéric II, en 1769, puis ambassadeur à Londres entre 1770 et 1776.

Tout le monde s'accordait à louer le talent de Guines pour la flûte, à commencer par Mozart lui-même : «Je crois vous avoir déjà écrit [...] que

(7) Ibidem, p. 256. Lettre du baron Grimm à Leopold Mozart, Paris, 21 février 1778.

(8) Ibid., p. 300-301. Lettre de Mozart à son père, Paris, $1^{\mathrm{er}}$ mai 1778. 
le duc de Guines [...] joue incomparablement de la flûte » ${ }^{9}$. Jouer de la flûte traversière supposait de bien posséder l'embouchure, ce qui ne se faisait guère aisément. L'inventaire après décès révèle l'intérêt du duc pour son instrument, puisqu'il ne mentionne pas moins de trois flûtes traversières pour un total de 126 francs en $1807^{10}$. À Berlin, il avait probablement entendu parler de Johann Joachim Quantz (1697-1773), musicien de la chambre du roi de Prusse, qui avait beaucoup fait pour perfectionner l'instrument et qui, en outre, était l'auteur d'une méthode réputée, parue en 1752. Le duc de Lévis laissait entendre que la passion partagée pour la flûte avec Frédéric II avait servi ses ambitions. En réalité, chez l'amateur, l'aristocrate n'était jamais loin.

Mêlant habilement le goût de la musique et sa pratique à un niveau élevé avec les stratégies de réussite sociale, il n'est pas douteux que ses activités musicales avaient contribué à renforcer ses liens avec MarieAntoinette, dont il devint l'un des intimes. Ainsi, en juin 1779, à Trianon, il fut en mesure de se mêler à deux musiciens professionnels de grand renom :

« [...] on lui donna le désir de descendre dans ses jardins. Là, elle fut surprise par les sons d'une musique céleste, [...] elle aperçut, dans une des niches du bosquet, un berger jouant de la flûte ; c'était M. le duc de Guines ; plus loin deux faunes, Begozzi et Ponto, qui exécutèrent un duo de cor et de hautbois, et, réunissant ensuite leurs accords avec ceux de la flûte, formèrent un trio charmant $»^{11}$.

Le duc passa commande à Mozart d'un concerto, celui pour flûte et harpe en ut majeur K. 299 (297c), et l'engagea comme maître de composition de sa fille aînée, également harpiste. À ce stade, Mozart semblait plutôt satisfait de son élève :

«Elle a un grand Talent, du génie et surtout une mémoire incomparable : elle joue tous les morceaux par cœur et en connaît certainement 200 [...]. Aujourd'hui, je lui ai donné la $4{ }^{\text {ème }}$ leçon, et en ce qui concerne les règles de la Composition et de l'harmonie, je suis assez content d'elle $»^{12}$.

(9) Ibid., vol. 2, p. 311. Post-scriptum de Mozart à son père, Paris, 14 mai 1778.

(10) AN, Minutier central, Étude LXIX/885, inventaire après de décès de M. Deguines de Bonnieres, 9 janvier 1807.

(11) Frédéric Melchior GRIMM, Correspondance littéraire, philosophique et critique, Paris, Tourneux, 1877-1882, vol. 12, p. 260-261.

(12) Wolfgang Amadeus MOZART, op. cit., vol. 2, p. 311. Post-scriptum de Mozart à son père, Paris, 14 mai 1778. 
Très vite pourtant, il se lassa et après avoir reconnu du génie à son élève, lâcha cet aveu d'impuissance : «Elle n'a aucune idée, il n'en sort rien. J'ai tout essayé avec elle $»^{13}$. Le découragement l'emportait, d'autant que son élève le laissa choir pour se marier. Pire encore, le duc ne lui paya pas le concerto et la totalité des vingt-quatre leçons professées. Mozart laissa alors exploser sa colère :

«La gouvernante tira une bourse et me dit : pardonnez-moi de ne vous payer que douze leçons pour cette fois, je n'ai pas assez d'argent ; - c'est noble ! [...] Mr le duc n'avait donc aucun honneur - et pensait : voici un jeune homme et de plus un Allemand benêt - comme disent les français qui s'en satisfera. Mais le nigaud d'Allemand ne fut pas satisfait - et n'accepta pas l'argent. [...] J'attends donc que le mariage soit passé puis j'irai chez la gouvernante et exigerai mon argent $[\ldots] \gg^{14}$.

Les désillusions étaient grandes, à la mesure des espoirs nourris par ce coûteux voyage. Elles s'ajoutaient au drame intime vécu par le jeune homme avec la disparition de sa mère, emportée par le typhus, le 3 juillet 1778. Le compositeur traversa une longue phase d'abattement dont les deux sonates en mode mineur pour piano et violon K. 304 (300c) et pour piano seul K. 310 (300d) portent incontestablement la marque.

Face à l'adversité, Mozart cherchait le réconfort auprès de ses amis musiciens de Mannheim également présents à Paris comme Wendling ou le ténor Anton Raaf. Il entra aussi en relation avec le comte Von Sickingen (1737-1791), envoyé du Palatinat à Paris, pianiste et compositeur amateur. Une véritable complicité lia tout de suite les deux hommes. Mozart se rendait régulièrement chez lui, y passait des journées entières à parcourir les partitions d'opéras du comte et à lui faire découvrir ses œuvres nouvelles comme la symphonie en ré majeur K. 297 (300a) dite « Parisienne » créée au Concert Spirituel le 18 juin 1778. Il trouva aussi un appui en la personne de la marquise Louise d'Épinay (1725-1783), compagne du baron Grimm et égérie des philosophes ${ }^{15}$. Elle entreprit de lui trouver un logement plus confortable et finit même par l'héberger dans son hôtel de la Chaussée d'Antin.

Durant l'été, alors qu'une vague de chaleur s'abattait sur le Bassin parisien et que l'aristocratie était à la campagne, Mozart fut invité chez le

(13) Ibidem.

(14) Ibid., vol. 3, p. 42. Lettre de Mozart à son père, 31 juillet 1778. 1995 , p. 961 .

(15) Jean de VigUERIE, Histoire et dictionnaire du temps des Lumières, Paris, R. Laffont, 
duc de Noailles (1713-1793) dans son château de Saint-Germain-en-Laye. Le duc de Noailles était un mécène de premier plan : ancien chanteur amateur des spectacles des «Petits Cabinets » sous l'égide de la marquise de Pompadour à la fin des années 1740, il était aussi un dédicataire recherché, l'un des souscripteurs de la Société Olympique et, surtout, entretenait un ensemble musical. Sous son patronage, Mozart passa dix jours agréables en compagnie des musiciens allemands du duc, du castrat italien Tenducci (1736-1790) et du plus jeune des fils de Johann Sebastian Bach, Johann Christian (1735-1782). Le témoignage de ce bain musical autant qu'amical dans lequel Mozart se plongea fut la composition de la scène dramatique pour Tenducci avec accompagnement de piano, hautbois, cor et basson K. 315b, laquelle n'est malheureusement pas parvenue jusqu'à nous.

Ce répit fut de courte durée. Sitôt revenu à Paris à la fin du mois d'août, Mozart se trouva aux prises avec le baron Grimm qui émettait des doutes sérieux sur ses capacités à réussir à Paris et décida d'en faire part à Léopold à l'insu du compositeur. Les deux hommes, l'un à Paris, l'autre à Salzbourg, les mêmes qui s'étaient engagés à tout faire pour sa réussite, commencèrent un siège méthodique pour ramener le musicien sur sa terre natale. La tension entre Mozart et Grimm devint très vive et provoqua la rancœur de Mozart : « Je lui ai ouvert mon cœur, comme à un véritable ami - et il en a fait usage ; il m'a toujours mal conseillé parce qu'il savait que je le suivrai $\gg^{16}$. En fait le désaccord se situait d'abord sur le terrain musical. Grimm, critique influent mais à la formation musicale superficielle, se heurtait à un compositeur de génie, conscient de l'être et très indépendant dans ses goûts. Aujourd'hui, les écrits de Grimm apparaissent contestables et bien souvent contradictoires. Son revirement le plus spectaculaire concerna l'œuvre de Rameau qu'il commença par trouver grande et originale en 1754 et qu'il jeta au caniveau dix ans plus tard : «Je veux mourir si Rameau et toutes ses notes ont jamais compté pour quelque chose dans le reste de l'Europe $\gg^{17}$. Il ne sut éviter le piège du pessimisme et du scepticisme, portant souvent la musique sur le terrain d'un affrontement entre nations, ce qu'il déplorait à d'autres moments.

L'examen de sa bibliothèque inventoriée au moment des saisies révolutionnaires révèle son goût quasiment exclusif pour l'opéra italien

(16) Wolfgang Amadeus MozART, op. cit., vol. 3, p. 80. Lettre de Mozart à son père, Paris, 11 septembre 1778 .

(17) Friedrich Melchior GRIMM, op. cit., vol. 6, p. 88. 
(près de $82 \%$ du total $)^{18}$. On comprend mieux alors l'exaspération de Mozart qui voyait en lui un godillot du «parti » italien.

Le 26 septembre 1778, c'est un Mozart écumant de rage, avec la sensation d'avoir été joué, qui refranchit, en sens inverse, les barrières de la ville. Ce départ précipité imposé par Grimm, lequel a été jusqu'à lui réserver une place dans la lente diligence de Strasbourg, scelle définitivement l'échec de ce troisième séjour parisien.

Nombre de musicologues se sont penchés sur ce rendez-vous manqué de Mozart avec la bonne société parisienne afin d'en rechercher les causes. Son caractère ombrageux ou son physique peu attirant ont ainsi été mis en avant. Mais que dire alors d'un Rameau ou d'un Gluck ? On a également insisté sur son fort accent tudesque, mais alors comment expliquer le succès de centaines de musiciens étrangers à Paris ? Dans le même registre, on a pointé du doigt son incapacité à adopter les usages de la sociabilité et de la mondanité aristocratiques. Qui pourtant mieux que lui, qui avait arpenté depuis sa tendre enfance toutes les cours européennes, pouvait en être le meilleur connaisseur ? Mozart était tout à fait capable de s'y adapter parfaitement, ainsi qu'il le dit lui-même : «Par ma complaisance, je me suis acquis amitié et protection ; si je devais tout écrire - j'aurai mal aux doigts $»^{19}$. On argue aussi le fait que Mozart n'aurait pas été lui-même, durant ces six mois, éloigné de celle dont il était tombé follement amoureux à Mannheim, la chanteuse Aloysia Weber. Mais alors, comment expliquer son regret maintes fois répété, jusqu'à un mois et demi après son départ, de quitter Paris précipitamment ?

Outre ces éléments imputés à Mozart lui-même, certaines analyses ont invoqué le contexte historique. Mozart aurait été ainsi la victime de la réaction aristocratique ${ }^{20}$. Le contexte musical est tout autant sollicité. La querelle des Gluckistes et des Piccinnistes, la concurrence avec les Cambini, Grétry et autres Gossec... auraient rendu impossible toute percée mozartienne. Sans se soucier du péril téléologique, des biographes ont énoncé les conclusions qui les arrangeaient jusqu'à affirmer que quitter Paris et retourner en Autriche était ce que Mozart avait à faire de mieux ${ }^{21}$.

(18) BNF, Département de la Musique. Inventaire manuscrit des musiques saisies chez les émigrés dressé par Bruni, achevé en juillet 1796, non côté.

(19) Wolfgang Amadeus MozART, op. cit., vol. 3, p. 79. Lettre de Mozart à son père, Paris, 11 septembre 1778 .

(20) Jean et Brigitte MAssin, Wolfgang Amadeus Mozart, Paris, Fayard, 1990 ( $1^{\text {re }}$ édition 1970), p. 236-237.

(21) Howard Chandler RobBins LANDON, « La place de Paris dans la vie et l'œuvre de Mozart », Mozart à Paris, Paris-Musées/Francis Van de Velde, 1991, p. 16. 
Mozart n'est resté que six mois à Paris. Sans véritable recommandation et sans y être invité, la probabilité de s'imposer dans un délai aussi court était faible. L'examen de la carrière de nombreux autres musiciens montre que la réussite supposait des séjours prolongés ou répétés. Ainsi Wendling s'était rendu deux fois à Paris et y revenait une troisième en 1778. Un autre compatriote allemand, Carl Stamitz, était arrivé en 1770, avait attendu plus d'un an pour devenir compositeur et chef d'orchestre au service du duc de Noailles et n'avait été reçu à Versailles qu'en 1772. Gluck, lui, était venu pour la première fois en 1764, avait multiplié les séjours en 1777 et 1779 . Piccinni, l'autre grand protagoniste de la scène lyrique, était arrivé en 1776 pour y demeurer jusqu'à sa mort en 1800 . Durant l'été 1778, sa correspondance montre de manière particulièrement insistante qu'il était décidé à faire montre de patience et qu'il commençait à entrevoir la possibilité de réussir :

« Dieu veuille qu'un changement intervienne bientôt ! - Entre-temps, je ne manquerai ni d'application, ni de peine ou de travail. Je mets quelque espoir dans l'hiver, lorsque tout le monde rentrera de la campagne $»^{22}$.

Mozart prenait progressivement conscience que sa venue se situait à contretemps du rythme musical parisien et que l'essentiel de la saison musicale se concentrait durant l'hiver, tant pour l'opéra que pour le concert ${ }^{23}$. Au début de septembre, l'horizon s'éclairait :

« Si je pouvais me décider à tenir bon ici quelques années, je ne doute pas d'être en mesure de vraiment bien arranger mes affaires. Je suis maintenant assez connu ici ; moi je ne connais pas les gens, mais eux savent qui je suis. Je me suis fait beaucoup d'honneur avec mes 2 symphonies [...]. Il m'aurait été maintenant possible de faire un opéra [...]. Toutefois, je vous dis franchement que mes affaires commençaient à bien marcher, il ne faut rien bousculer; chi va piano, va sano. Par ma complaisance, je me sui acquis amitié et protection ; si je devais tout écrire - j'aurais mal aux doigts $[\ldots] »^{24}$.

(22) Wolfgang Amadeus MozART, op. cit., vol. 3, p. 79. Lettre de Mozart à son père, Paris, 31 juillet 1778.

(23) Nous renvoyons le lecteur aux analyses de Joann Élart sur le calendrier des concerts parisiens. « Retour sur le séjour de Mozart à Paris en 1778 », Mozart et la France, de l'enfant prodige au génie (1764-1830), ouvrage coordonné par Jean GRIBENSKI et Patrick TAÏEB, Lyon, Symétrie, 2015 [à paraître], p. 21-38. Nous ne souscrivons cependant pas à son ultime conclusion qui affirme que Mozart ignorait les logiques de production dans l'espace public.

(24) Wolfgang Amadeus MOZART, op . cit., vol. 3, p. 79. Lettre de Mozart à son père, Paris, 11 septembre 1778 . 
Une chose est sûre : Mozart était sincère. Tout enfiévré qu'il était à la pensée de revoir Aloysia Weber en repassant par Mannheim, il accordait cependant la priorité à sa réussite parisienne. Mozart eut clairement le sentiment de faire le mauvais choix en quittant Paris. D'ailleurs, ce n'est pas tout, il envisageait de demeurer à Paris plusieurs années, sans abdiquer son regard sévère sur la France en général et Paris en particulier. Le 15 octobre 1778, il écrivait à son père :

«Et j'ai pensé en moi-même que si je n'avais pas eu de tels ennuis dans la maison où je logeais et que si les choses ne s'étaient pas précipitées avec la rapidité de l'orage, $\mathrm{j}$ 'aurais finalement pu réfléchir calmement à toute cette affaire - je vous aurais prié d'avoir encore un peu de patience et de me laisser encore à Paris. Je vous assure que je serais parvenu à y obtenir honneur, gloire et argent - et vous aurais sûrement débarrassé de vos dettes $»^{25}$.

Quinze jours plus tard, il ne décolérait pas, n'ayant pu achever ses affaires en $\operatorname{cours}^{26}$.

L'examen de l'ensemble des démarches entreprises par Mozart durant ces six mois montre que sans réelle stratégie préétablie, il réussit néanmoins à faire entendre ses compositions à l'Académie royale de musique, au Concert Spirituel, à se voir commander des œuvres par des patrons importants, à avoir trois élèves et à faire graver ses œuvres ${ }^{27}$. Mieux encore, son nom apparaît seize fois entre 1778 et 1789 dans les programmes du Concert Spirituel et une cinquantaine d'œuvres sont publiées à Paris de son vivant, dont une quinzaine en première édition ${ }^{28}$. Son réseau relationnel était déjà étoffé : nobles allemands à Paris (Sickingen), univers des Encyclopédistes (Grimm, Marquise d'Épinay), aristocratie de cour (Guines, Noailles, Rohan-Chabot).

En fin de compte, son échec résulte bien davantage de la trop grande brièveté de son séjour, abdiquant du même coup sa propre volonté face aux injonctions de Grimm et de son père, lequel n'hésitait pas à le rendre responsable de la mort de sa mère, que de l'idée infondée qu'il n'y avait

(25) Ibidem, p. 96. Lettre de Mozart à son père, Strasbourg, 15 octobre 1778.

(26) Ibid., p. 102. Lettre de Mozart à son père, Strasbourg, 2 novembre 1778.

(27) Mozart n'obtint pas de poste permanent, mais le pouvait-il, lui qui ne voulait rien d'autre qu'un poste de maître de chapelle ? Il fit état en mai d'un poste d'organiste à Versailles que lui aurait proposé le corniste Johan Joseph Rudolph, musicien de la Chapelle royale. Mozart écarta la proposition, fût-elle vague ou sérieuse. Olms, 2006.

(28) Jean GRIBENSKI, Catalogue des éditions françaises de Mozart 1764-1825, Hildesheim, 
pas de place pour lui à Paris. La meilleure preuve nous en est donnée quatre ans plus tard, en 1782 : «Mon idée est d'aller à Paris pour le prochain carême ; bien entendu pas au petit bonheur $»^{29}$. Ce nouveau voyage ne vit pas le jour. Mozart ne revint jamais en France. Mais à l'évidence, Paris et ses ressources musicales restaient gravées dans sa mémoire.

Depuis Paris, où ses premières sonates éditées furent dédiées à Victoire de France, jusqu'à Vienne, où sa mort interrompit la genèse du Requiem commandé par le comte Walsegg, la musique de Mozart fut l'incarnation désirée autant que nécessaire de la société aristocratique, bien avant que de devenir le sommet olympien de la culture européenne.

David HENNEBELLE Centre de Recherche et d'Études Histoire et sociétés Université d'Artois 9, rue du Temple BP 10665 62030 ARRAS cedex Hennebelle.David@wanadoo.fr 